$\mathrm{e}^{+} \mathrm{e}^{-}$Collisions from Phi to Psi 2013 (PHIPSI2013)

International Journal of Modern Physics: Conference Series

Vol. 35 (2014) 1460392 (7 pages)

(C) The Author

DOI: $10.1142 /$ S2010194514603925

\title{
DARK FORCES SEARCHES IN FIXED TARGET EXPERIMENTS
}

\author{
M. BATTAGLIERI \\ Istituto Nazionale di Fisica Nucleare, Sezione di Genova, \\ Via Dodecaneso 33, 16141 - Genova, Italy \\ battaglieri@ge.infn.it
}

Published 18 December 2014

\begin{abstract}
Searches for physics Beyond the Standard Model (BSM) can be carried out with precise and GeV-energy-range experiments. In many string theories, a Hidden Sector, decoupled to the SM, foresees the existence of a new massive boson, the $A^{\prime}$ or heavy photon, that weakly couples to the electromagnetic current. A new particle with mass in the range of $1 \mathrm{MeV}$ - $1 \mathrm{GeV}$ could explain many astro-particle observations (e.g. positron excess seen by PAMELA and AMS experiments) and some anomalies not yet fully understood (e.g. muon $g-2$ factor). The search for $A^{\prime}$ has motivated intense experimental activities in almost every accelerator facility using different techniques: colliding beam, fixed target experiments, meson rare decays. Jefferson Lab, a world-leading nuclear physics laboratory, is planning a set of fixed target experiments aiming to discover the $A^{\prime}$ or set new limits in its mass and coupling, with an unprecedented sensitivity and reach capability. In this contribution, after reviewing the physics case and some experimental evidences, I will report on the program of measurements planned at Jefferson Lab for the next years.
\end{abstract}

Keywords: Hidden sector; Heavy photon; Dark Matter; Beam dump experiments.

\section{Introduction}

Experiments carried at the Energy Frontier just discovered the Higgs boson, further confirming the Standard Model of elementary particles. First investigations of rare processes, e.g. $B-$ meson rare decays, showed no hints of new strongly interacting particles or heavy electro-weak bosons. The lack of signals of new physics beyond the Standard Model, demands to be addressed our research in other directions too. What if new physics could manifest in limited energy range (around the proton mass) but with extremely weak coupling, e.g. in the range $\left(10^{-4}-10^{-11}\right) \times \alpha_{E M}$ ? In the last decades access to high luminosity machine and novel detection technologies enabled searches for new physics at otherwise well-explored energies. ${ }^{1}$ At the

This is an Open Access article published by World Scientific Publishing Company. It is distributed under the terms of the Creative Commons Attribution 3.0 (CC-BY) License. Further distribution of this work is permitted, provided the original work is properly cited. 
Intensity Frontier, searches for new physics often rely on the study of rare processes and the search for subtle effects which would indirectly indicate physics beyond the Standard Model. But this is not the rule. New studies of otherwise commonplace phenomena at electron machines, like trident production on heavy nuclear targets, can, with sufficient sensitivity, explore whole new worlds and directly search for hidden sector particles and forces, those without direct couplings to our Standard Model world.

\section{Physics case}

In nature there are four known fundamental interactions: gravitational, electromagnetic, weak and strong. Each force has an associated gauge-boson that mediates the interaction between particles carrying the related charge. There are several indications pointing to the existence of another kind of particles, not yet detected, forming the so called Dark Matter. This interacts very weakly with normal matter and may account for up a quarter of the universal mass-energy. The Dark Matter may inhabit a Hidden Sector and interact very weakly with normal, baryonic matter. This new sector could include complex new forces and other new particles with which we barely interact. Is indeed quite natural to conceive a new fundamental force that mediates the dark matter interaction and that manifests itself via the existence of a new gauge-boson. Heavy photons, or Dark or Hidden Sector photons, may well be this new gauge bosons, being part of our universe and related to Dark Matter. ${ }^{2}$ The observation of very high energy positrons reported by PAMELA ${ }^{3}$ and AMS $^{4}$ experiments is difficult to be understood in terms of SUSY dark matter annihilation. Some authors reconcile the observed flux by proposing models where massive dark matter particles annihilate to hidden sector photons, which in turn decay to high energy electron-positron pairs. ${ }^{5}$ These theories presume heavy photons couple to dark matter, mediate its interactions, are produced in its annihilation, and weakly couple to electric charge. Heavy photons in the mass range of 20 to $1000 \mathrm{MeV}$ can reasonably account for the observed cosmic ray fluxes. From the theoretical point of view, many BSM theories include an extra U(1) gauge group whose associated gauge-boson may have mass over a very wide range. Such heavy photons may kinetically mix with our own photon, leading to their induced coupling to electric charge. This mixing can be mediated by GUT level particles which carry both Standard Model hypercharge and its hidden sector analogue. Interestingly, the natural scale for this mixing results in heavy photons coupling to Standard Model charged particles with couplings of order $10^{-3} e$. Heavy photons naturally couple to electrons, albeit with couplings much suppressed when compared to those in standard QED. It follows that electrons will radiate heavy photons, and heavy photons will decay to electron-positron pairs or pairs of other kinematically accessible charged particles, but at rates significantly below QED trident production, and with lifetimes far longer than those expected from purely electromagnetic interactions. Other consequences of the proposed mechanisms should lead to a local modification of the 
electromagnetic interaction. The observed deviation from SM prediction for $(g-2)_{\mu}$ or the discrepancy in the proton radius value when measured by the muonic hydrogen Lamb shift could be explained by invoking the existence of the heavy photon. ${ }^{6}$

\section{Experimental searches}

The experimental searches for a new massive gauge boson span many orders of magnitudes in mass. Axion-like particles with $\mathrm{M} \leq 1 \mathrm{MeV}$ (down to $10^{-15} \mathrm{eV}$ ) were searched for with different techniques, providing stringent upper limits in the Coupling vs. Mass parameter space. High energy experiments at LHC are testing the high mass range with limited sensitivity for small couplings. In between, many medium energy facilities in the world, Jefferson Lab, SLAC and Fermi Lab in the USA, Mainz in Germany, DAPHNE in Italy, and Serpukhov in Russia, are analysing data collected for other purposes to identify the $A^{\prime}$ in lepton-pair final states. Intense experimental activity is foreseen for the near future with proposals that optimize the running conditions of dedicated runs. In the following I will only review the measurement program planned at Jefferson Lab, as an example of the new generation of fixed target experiments. Parallel searches at colliders (Babar, Belle, Kloe) deserve a thorough discussion that is outside the scope of these proceedings.

\subsection{Fixed target experiments}

In a fixed target experiment, a few-to-several Gev electron beam is shot over a high-Z target to provide a sizeable number of Bremsstrahlung photons irradiated by the nuclear field. ${ }^{7}$ Photons can convert to an $A^{\prime}$ that in turn decays to an $e^{+} e^{-}$ pair detected by an apparatus placed downstream the target. The experimental signature of the new particle is a narrow bump, peaked at the mass of the $A^{\prime}$ over an irreducible background due to trident production and Bethe-Heitler processes. This technique is usually referred to as bump hunting. If the target is thick (several radiation lengths), photons are dumped and only long-lived particles, such as the $A^{\prime}$, cross the dump, decaying in the instrumented area. The detector acceptance is related to the mass range sensitivity, determining the available phase space in the decay. A trade off between the target length, long enough to dump the background but short enough to provide a measurable decay rate, and the size of the instrumented area allows one to explore the widest range of the parameter space. This idea was applied in the '80s, setting upper limits reported in Fig. 1. The new experiments, taking advantage of improved detection technology, increase the resolution in peak mass determination, and therefore the sensitivity of an upper limit in case of negative founding. Together with the bumphunting technique, novel experiments use the vertexing capability provided by increased control of beam parameters and silicon detector technology, which are able to work in a high background environment. A detached vertex, far from a spot-like beam-target interaction point, allows identification of a long-lived resonance and, correspondingly, test of the low coupling zone at sizeable $A^{\prime}$ mass. Bump hunting and vertexing together provide an 


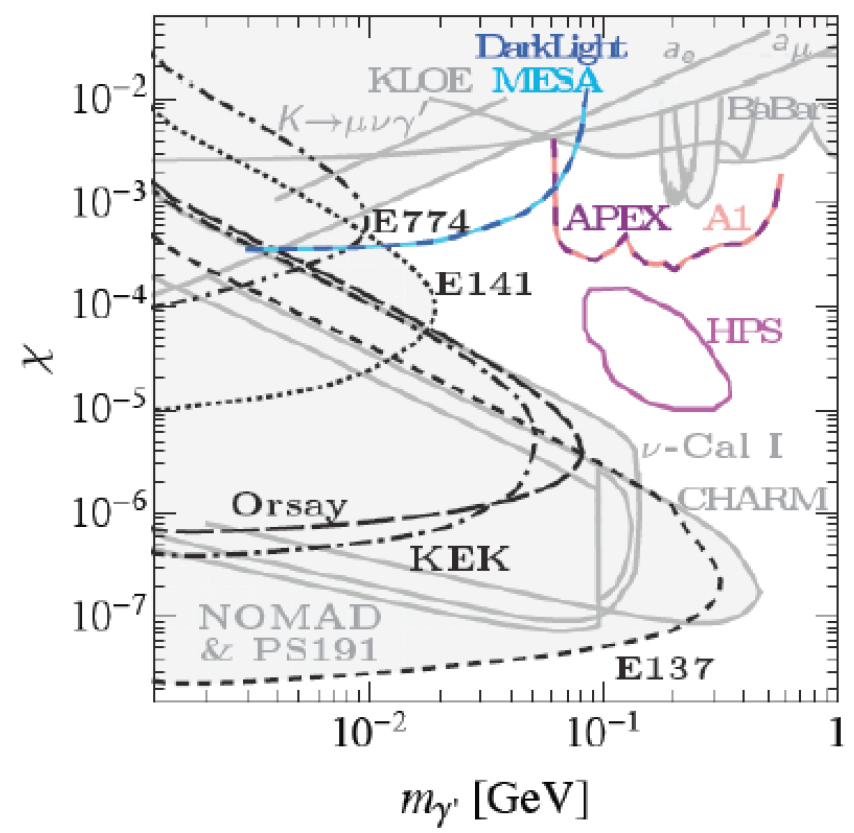

Fig. 1. The limits on $A^{\prime}$ set by previous experiments in the Coupling vs. Mass parameters space. The expected reach of APEX, HPS and DARK LIGHT experiments planned at Jefferson Lab are also shown.

extended sensitivity to unexplored regions of the parameter space. See again Fig. 1 where the reach expected by experiments planned for the near future at Jefferson Lab. In the following I will briefly describe the Heavy Photon Search experiment or $\mathrm{HPS}^{8}$ that, together with A-Prime Experiment or $\mathrm{APEX}^{9}$ and Dark Light, ${ }^{10}$ are planned at Jefferson Lab and will apply these techniques.

\section{Jefferson Lab and the HPS experiment}

Jefferson Lab, located in Newport News Virginia (US), hosts CEBAF (Continuous Electron Beam Accelerator Facility) an electron accelerator facility mainly devoted to study hadron physics. It has been operational for more than 15 years running an up-to $6 \mathrm{GeV}$ highly polarized electron beam to the three experimental Halls (A-B and $\mathrm{C}$ ), equipped with high-precision magnetic spectrometers (Hall A and C) and a $4 \pi$ detector called CLAS (CEBAF Large Spectrometer). It is now under a major upgrade that will bring the maximum beam energy up to $12 \mathrm{GeV}$, add a new experimental Hall (Hall-D) and renew the detector equipment in the existing Halls. An almost continuous-wave beam structure together with excellent beam characteristic in term of energy and position spread are the key-elements in enlarging the original physics program to include precision experiments sensitive to physics BSM.

The Heavy Photon Search (HPS) is an experiment proposed for Hall-B at Jefferson Lab to search for new heavy vector boson(s), in the mass range of $20 \mathrm{MeV} / \mathrm{c}^{2}$ to 


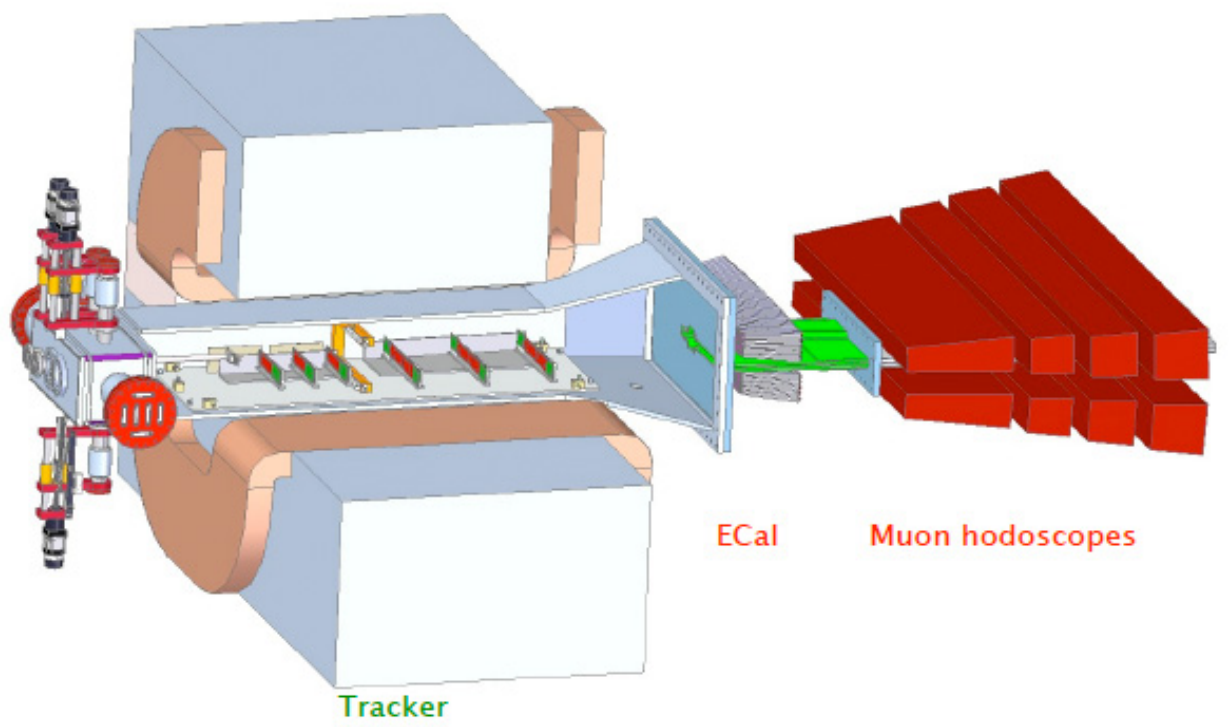

\section{Measurement}

\section{Trigger and PID}

Fig. 2. The HPS detector components. From left to right: the target, the silicon tracker in the dipole magnet, the electromagnetic calorimeter ECal and the muon detecor.

$1000 \mathrm{MeV} / \mathrm{c}^{2}$. HPS searches for electro-produced heavy photons using both invariant mass (bump hunting) and separated decay vertex signatures (vertexing), using a compact, large acceptance forward spectrometer. HPS will utilize a setup based on a three-magnet chicane, the second dipole magnet serving as the analyzing magnet for the forward spectrometer. The detector package, shown in Fig. 2, will include a silicon tracker, an electromagnetic calorimeter, and a muon detector. High luminosities are needed to search for heavy photons with small couplings and masses in the 20 to $1000 \mathrm{MeV}$ range. Utilizing CEBAF essentially continuous duty cycle, the experiment can simultaneously maximize luminosity and minimize backgrounds by employing detectors with fast response and rapid readout. The HPS setup is designed to run with $>200 \mathrm{nA}$ electron beams at energies from $1.1 \mathrm{GeV}$ to $6.6 \mathrm{GeV}$ impinging on a tungsten target.

The HPS tracker consists of six double layer planes, 36 microstrip sensors in total. Placing the planes of the tracker in close proximity to the target means that the primary beam must pass directly through the middle of the tracking detector. This has necessitated for the sensors not to encroach on a dead zone, where multiple Coulomb scattered beam particles and radiative secondaries are bent into the horizontal plane. However, since the energy released in the decay of a low mass $\mathrm{A}^{\prime}$ is small relative to its boost, the opening angle between decay daughters can be quite small. To maximize the acceptance for low mass $\mathrm{A}^{\prime} \mathrm{s}$, the vertical extent of the 
dead zone must be minimized and sensors placed as close as possible to the beam. To minimize production of secondaries on air, the entire tracking and vertexing system is placed in vacuum. Silicon microstrip sensors are used in the tracker/vertexer because they collect ionization in tens of nanoseconds and produce pulses as short as $50-100 \mathrm{~ns}$. The sensors are read out continuously at $40 \mathrm{MHz}$ using the APV25 chip, developed for the CMS experiment at the LHC.

A lead-tungstate electromagnetic calorimeter, with APD readout, is used to trigger the DAQ. Like the tracker system, the electromagnetic calorimeter is split into an upper and a lower part to avoid impinging on the dead zone. The beam and radiative secondaries pass between the upper and lower ECal modules, which are housed in temperature-controlled enclosures, needed to stabilize the energy calibration.

The muon detection system will be installed downstream of the ECal, which has absorbed most of the electromagnetic background produced in the target. The remaining backgrounds will be attenuated by the first absorber layer of the muon system. The muon system will consist of four double layers of scintillator hodoscopes sandwiched between iron absorbers. Light from scintillator strips will be transported to photo-multiplier tubes via wave-length shifting fibers embedded within the strips. As in case of the ECal, the muon system will be divided into two parts, beam up and beam down.

Commissioning of HPS is scheduled in the Fall of 2014 and data are expected to be to taken starting from Spring 2015.

\section{Conclusions}

In recent years, the physics community realised that experiments at the Intensity Frontier can be run to search for physics BSM. In particular, a new generation of beam dump experiments that use state-of-the art detector technologies and exploit the progresses achieved by accelerated beams will be able to put stringent constraints in the parameter space looking for new particles weakly coupled to the SM. The are some theoretically-motivated scenarios that predict the existence of a new gauge boson called Dark Photon or $A^{\prime}$ that can be produced in photon-rich environments such as radiation of a multi-GeV energy electron in a high $\mathrm{Z}$ nuclear field. The existence of the $A^{\prime}$ could also reconcile the astrophysical observation of an excess of high energy positrons and some unexplained discrepancies in electro-magnetic properties of leptons. A comprehensive program that include experiments in different experimental Halls is planned to run at JLab in the near future. The Heavy Photon Search experiment in Hall-B, the A-Prime-Experiment in Hall-A and the Dark Light experiment at the Jefferson Lab Free Electron Laser will take data with an unprecedented reach capability, providing stringent constraints in unexplored regions of the Coupling vs Mass parameter space.

\section{References}

1. Dark2012: Dark Forces at Accelerators, http://www.lnf.infn.it/conference/dark/ index.php 
2. B. Holdom, Phys. Lett. B 166 (1986) 196.

3. O. Adriani et al. [PAMELA Collaboration], Phys. Rev. Lett. 106, 201101 (2011) [arXiv:1103.2880 [astro-ph.HE]].

4. M. Aguilar et al. [AMS Collaboration] Phys. Rev. Lett. 110, 141102 (2013).

5. M. Pospelov and A. Ritz, Phys. Lett. B 671 (2009) 391 [arXiv:0810.1502 [hep-ph]].

6. M. Pospelov, Phys. Rev. D 80 (2009) 095002 [arXiv:0811.1030 [hep-ph]].

7. J. D. Bjorken, R. Essig, P. Schuster and N. Toro, Phys. Rev. D 80, 075018 (2009) [arXiv:0906.0580 [hep-ph]].

8. P. Hansson Adrian et al. https://confluence.slac.stanford.edu/download/attachments/ 86676777/hps_2014.pdf?version=1\&modificationDate=1368227154000\&api=v2

9. S. Abrahamyan et al. [APEX Collaboration], Phys. Rev. Lett. 107, 191804 (2011) [arXiv:1108.2750 [hep-ex]].

10. M. Freytsis, G. Ovanesyan and J. Thaler, JHEP 1001 (2010) 111 [arXiv:0909.2862 [hep-ph]]. 